\title{
A(s) casa(s) e o "ficar em casa": Um estudo exploratório dos impactos psicossociais das desigualdades habitacionais na vivência da pandemia da Covid-19
}

\begin{abstract}
Raquel Ribeiro'
${ }^{1}$ Centro de Estudos Sociais, Universidade de Coimbra, Portugal | raquelribeiro@ces.uc.pt | https://orcid.org/0000-0003-4113-0664

Resumo: $O$ direito à habitação é inseparável do direito à vida e constitui a base de outros direitos humanos fundamentais. A pandemia da Covid-19 colocou a habitação na primeira linha de defesa contra o novo coronavírus tornando mais visíveis e dramáticas as desigualdades estruturais na habitação. Este artigo pretende estudar o impacto das condições habitacionais na vivência da pandemia da Covid-19 recorrendo a métodos mistos. Apresenta os resultados preliminares de um inquérito por questionário com perguntas abertas e fechadas aplicado a 527 residentes em Portugal em 2021. Os resultados revelam que as pessoas com melhores condições habitacionais reportam um impacto mais positivo da habitação, nomeadamente na saúde física e psicológica durante a pandemia. A análise textual com o software IRAMUTEQ às respostas à questão aberta revela que o impacto positivo se associa sobretudo à possibilidade de praticar exercício físico e de desenvolver atividades de lazer ao ar livre. Pelo contrário, a falta de espaço interior ou exterior, os problemas de humidade e infiltrações, o mau isolamento sonoro ou a pouca exposição solar estão associados à experiência de problemas de saúde e deterioração do bem-estar emocional e psicológico. Os resultados sugerem que a adesão às medidas de confinamento e isolamento social em casa produzem vivências muito diferenciadas em função das condições habitacionais das populações, com importantes impactos quer ao nível da saúde física quer da saúde mental. Conclui-se da necessidade de combinar metodologias quantitativas e qualitativas na compreensão da relação entre as desigualdades nas condições habitacionais e os impactos psicossociais da pandemia da Covid-19.
\end{abstract}

Palavras-chave: Habitação; Pandemia da Covid-19; Desigualdades Sociais; Impactos Psicossociais; Métodos Mistos.

Home(S) and "Staying at Home": An Exploratory Study of the Psychosocial Impacts of Housing Inequalities During the Covid-19 Pandemic

Abstract: The right to housing is inseparable from the right to life and constitutes the basis for other fundamental human rights. The Covid-19 pandemic put housing at the forefront of defence against the new coronavirus, making structural inequalities in housing more visible and dramatic. This article aims to study the impact of housing conditions on the Covid-19 pandemic experience using mixed methods. It presents the preliminary results of a survey with open and closed questions applied to 527 residents in Portugal in 2021. The results show that people with better housing conditions report a more positive impact of housing during the pandemic, namely on physical and psychological health. The textual analysis with the software IRAMUTEQ to the answers to the open question reveals that the positive impact is associated mainly with the possibility of practicing physical exercise and developing leisure activities outdoors. On the contrary, the lack of indoor or outdoor space, problems with humidity and infiltration, poor sound insulation, or little sun exposure are associated with the experience of health problems and deterioration of emotional and psychological well-being. The results, therefore, suggest that the adherence to the measures of confinement and social isolation at home produce very differentiated experiences depending on the housing conditions of the populations, with important impacts both in terms of physical and mental health. It is concluded that there is a need to combine quantitative and qualitative methodologies to understand the relationship between inequalities in housing conditions and the psychosocial impacts of the Covid-19 pandemic.

Keywords: Housing; Covid-19 Pandemic; Social Inequalities; Psychosocial Impacts; Mixed Methods. 


\section{Introdução}

O direito à habitação é inseparável do direito à vida (Farha, 2016) e constitui a base de outros direitos humanos fundamentais como a saúde e a educação. A pandemia da Covid19 provocada pelo novo coronavírus (ONU, 2020) colocou a habitação na primeira linha de defesa contra o vírus - associada ao lema "fique em casa" - e tornou não só mais visíveis as desigualdades estruturais na habitação como mais pungentes (Farha, 2020).

A provisão da habitação tem estado historicamente associada à (re)produção de desigualdades sociais, económicas e territoriais (Harvey, 1973), contudo estas agravaramse à medida que os mercados, atores e motivações financeiras adquiriram preponderância na sua provisão e a propriedade habitacional passou a ser utilizada como um ativo financeiro para investimento (Rolnik, 2013). Este processo de financeirização da habitação (Aalbers, 2016) tem tido especial incidência nos centros urbanos onde a pressão especulativa é maior (Fernandez, Hofman, \& Aalbers, 2016) e onde se verifica uma maior e mais profunda inter-relação entre construção urbana, crédito à habitação e concentração das atividades financeiras (Ribeiro \& Santos, 2019). Os impactos incluem o aumento das desigualdades no acesso à habitação e nas condições de habitabilidade, nomeadamente entre proprietários e arrendatários, aumento da insegurança habitacional e empobrecimento das populações menos favorecidas (Santos, 2019). Com mais da metade da população mundial a viver em áreas urbanas, as desigualdades na habitação estão no centro dos desafios enfrentados pelas sociedades contemporâneas para alcançar maior igualdade social, inclusão, cidadania plena e uma vida saudável (Alexander, Bruun, \& Koch, 2018).

As medidas preconizadas por muitos países, incluindo Portugal, para enfrentar a pandemia provocada pelo novo coronavírus incluíram um conjunto de diretrizes e restrições com vista a diminuir a circulação e o contacto entre pessoas. Os dois períodos de maior confinamento (genericamente de março a maio de 2020 e de janeiro a março de 20211) implicaram, entre outros, o encerramento de estabelecimentos comerciais não essenciais, do atendimento em diversos serviços públicos e dos estabelecimentos de ensino; o trabalho a partir de casa (teletrabalho) sempre que possível; e a proibição de permanência no espaço público, nomeadamente em jardins, parques infantis, etc., confinando a população ao espaço da sua habitação.

Neste artigo exploramos o impacto das condições habitacionais na vivência das alterações provocadas pela pandemia da Covid-19 através de métodos mistos. Mais concretamente, procurámos responder às seguintes questões de investigação (QI):

Q11: As condições habitacionais produzem um impacto diferenciado na vivência da pandemia da Covid-19?

Q12a: Que dimensões representacionais da casa têm influência na vivência da pandemia da Covid-19?

Q12b: As diferentes dimensões representacionais da casa expressam posições ancoradas no tipo de habitação, nas condições habitacionais ou no regime de ocupação dos respondentes (i.e. tratar-se de habitação própria, arrendada ou outra situação)?

Para o efeito procedeu-se à análise das respostas preliminares a um inquérito por questionário realizado em Portugal durante o segundo período de confinamento generalizado.

1 As restrições e medidas aplicadas pelo Governo Português podem ser consultadas em: https://dre.pt/legislacao-covid-19-por-data-de-publicacao\#32020 


\section{Métodos}

\subsection{Instrumento e Procedimento}

O estudo foi realizado através de inquérito por questionário aplicado à população residente no território português continental e insular. O questionário era constituído por 52 grupos de perguntas de resposta aberta e fechada destinadas a conhecer a opinião das pessoas sobre alguns aspetos da pandemia da Covid-19 e recolher informação sobre alguns fatores, como a habitação, onde os seus impactos psicossociais se podem fazer sentir (Ribeiro et al., 2021).

A primeira página do questionário apresentava os objetivos do estudo, garantia o anonimato e a confidencialidade das informações recolhidas e solicitava o consentimento informado à participação. Para o propósito do presente estudo iremos deter-nos na análise dos grupos de questões relativos aos problemas habitacionais e ao impacto da casa (pelas suas características de espaço, conforto ou pelo número ou tipo de problemas) na forma como os respondentes têm vivenciado as alterações provocadas pela pandemia da Covid19.

O primeiro grupo de questões pedia para indicar com "sim" ou "não" os problemas apresentados pela casa onde reside, de uma lista de dez: ausência de sanita com autoclismo dentro de casa; ausência de banheira ou de chuveiro; janelas, portas ou chão podres; humidade ou infiltrações nas paredes ou nos tetos; falta de instalações de aquecimento adequadas; falta de espaço; pouca luz natural; falta de lugar para estar no exterior (ex. um jardim, uma varanda, um terraço); mau isolamento sonoro; ou má ventilação.

O segundo grupo de questões incluía cinco perguntas de resposta fechada através de escala de opinião de 7 pontos relativas ao impacto (1=impacto muito negativo; 7 =impacto muito positivo) da casa onde vive na vivência das alterações provocadas pela pandemia da COVID-19 na saúde física, no bem-estar emocional e psicológico, na atividade profissional, na educação das crianças/jovens (no caso de viver com crianças/jovens) e na adesão às medidas de confinamento e isolamento social; e uma pergunta de resposta aberta: "Por favor, dê exemplos de como a casa onde vive tem influenciado a sua vida desde o início da pandemia".

A recolha dos dados decorreu online através da ferramenta Limesurvey, entre 12 de fevereiro e 15 de março de 2021. Para divulgar o inquérito utilizou-se a estratégia da bola de neve e divulgação, via email, junto de diversas instituições nacionais (universidades: órgãos municipais e de juntas de freguesia; associações sem fins lucrativos de natureza diversa).

\subsection{Participantes}

Participaram no inquérito 527 pessoas, na sua maioria do sexo feminino $(75.7 \%)$, com idades compreendidas entre os 18 e os 75 anos de idade $(M=45.08$; $D P=10.85)$ e residentes nas diversas regiões de Portugal $(17.1 \%$ no Norte, $18.9 \%$ no Centro, $21.1 \%$ na Área Metropolitana de Lisboa, $8.0 \%$ no Alentejo, $19.6 \%$ no Algarve, $14.6 \%$ nos Açores e $0.8 \%$ na Madeira). 
No que refere ao tipo de habitação, $49.7 \%$ reside em moradia, $47.8 \%$ em apartamento e $2.5 \%$ noutras situações (e.g. quarto). Relativamente ao regime de ocupação, a maioria dos participantes é proprietário da habitação onde reside (73.9\%), sendo que $57.4 \%$ adquiriu a habitação com recurso ao crédito bancário e $16.5 \%$ através de outros meios (poupança, herança, empréstimo familiar). No regime de arrendamento privado encontrase $14.7 \%$ e $1.6 \%$ no regime de renda apoiada. Ainda, $8.6 \%$ vive em habitação cedida gratuitamente e $1.2 \%$ noutras situações (habitação precária, quartos). A Figura 1 apresenta a distribuição dos problemas habitacionais reportados em função do regime de ocupação.

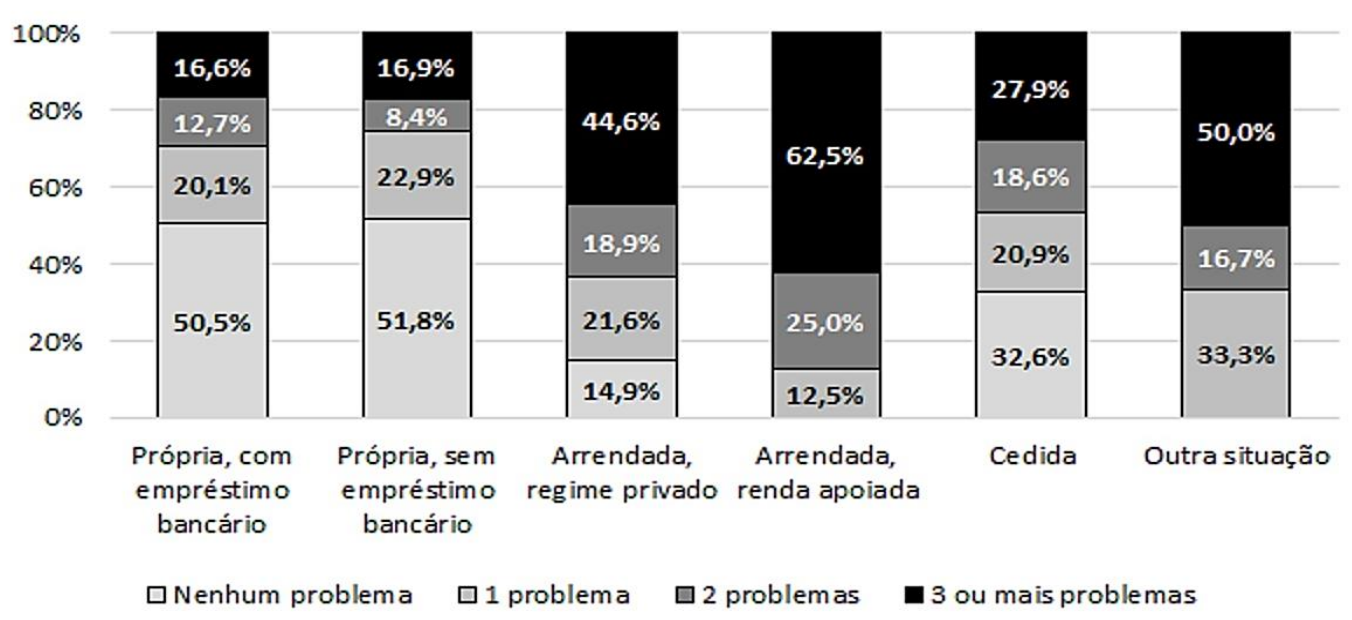

Fig. 1. Distribuição dos problemas habitacionais em função do regime de ocupação.

\subsection{Análise dos Dados}

Para caracterizar a amostra e responder à Q11 "As condições habitacionais produzem um impacto diferenciado na vivência da pandemia da Covid-19?" recorreu-se ao software SPSS (Statistical Package for Social Science for Windows, versão 25.0). Primeiro, procedeu-se à análise dos problemas da casa reportados pelas pessoas participantes criando uma variável relativa aos problemas habitacionais com quatro categorias: nenhum problema, um problema, dois problemas, três ou mais problemas. Posteriormente, para avaliar o impacto diferenciado na vivência da pandemia da Covid-19 nas diferentes esferas (saúde física, bem-estar, atividade profissional, educação das crianças/jovens e adesão às medidas de confinamento) foram realizadas análises de variância considerando a variável problemas habitacionais como variável independente e usando o teste de Bonferroni para comparações múltiplas sempre que encontrados efeitos positivos. No caso de violação do pressuposto de homogeneidade das variâncias foi aplicada a correção de Welch e o teste de Games-Howell para avaliar as diferenças entre grupos.

Para responder à questão de investigação Q12a "Que dimensões representacionais da casa têm influência na vivência da pandemia da Covid-19?" aplicou-se o método ALCESTE (Reinert, 1990) com o programa de análise textual IRAMUTEQ (Ratinaud, 2009) ao corpus textual correspondente às respostas à questão aberta "Por favor, dê exemplos de como a casa onde vive tem influenciado a sua vida desde o início da pandemia". Este método tem a vantagem de permitir identificar, de forma relativamente rápida, diferentes dimensões num dado corpus textual auxiliando o investigador na interpretação qualitativa dos mesmos (e.g. Ramos, Lima, \& Amaral-Rosa, 2018). Ao analisar a estrutura formal da coocorrência de palavras num dado corpus possibilita a identificação de diferentes "mundos lexicais" (Reinert, 1993), extraindo classes de palavras que apresentam vocabulário semelhante entre si e se diferenciam de outras classes de palavras coocorrentes. 
A interpretação qualitativa das classes de palavras extraídas pela análise possibilita identificar as dimensões representacionais da casa identificadas pelas pessoas participantes como tendo influência na vivência da pandemia da Covid-19 e desta forma responder à questão de investigação.

A possibilidade de introduzir variáveis suplementares na análise, como o tipo de habitação, o número de problemas identificados na habitação e o regime de ocupação, permite associar as dimensões representacionais a grupos particulares de participantes e assim responder à questão de investigação Q12b "As diferentes dimensões representacionais da casa expressam posições ancoradas no tipo de habitação, nas condições habitacionais ou no regime de ocupação dos respondentes?"

\section{Resultados}

Os resultados das análises de variância revelam diferenças estatisticamente significativas no que refere ao impacto das condições habitacionais, avaliado pelo número de problemas reportado, na vivência da pandemia (ver Tabela 1). As pessoas que residem em habitações sem problemas reportam um impacto bastante positivo em todas as esferas questionadas enquanto que as pessoas que residem em habitações com três ou mais problemas são aquelas que avaliam o impacto da habitação como menos positivo.

A análise textual com o método ALCESTE realizada sobre o corpus de palavras evocadas em resposta à questão aberta incidiu sobre 242 unidades de texto (285 pessoas não responderam à questão aberta 2 ). As 242 unidades de texto correspondem a 1154 formas e incluem todas as palavras com frequência mínima de 3 ocorrências. Da análise foram extraídas quatro classes de palavras coocorrentes, tendo sido classificadas $85 \%$ das formas (ver Figura 2).

Tabela 1. Impacto da habitação em função do número de problemas habitacionais. Médias (1=impacto muito negativo; 7 =impacto muito positivo).

\begin{tabular}{lccccc}
\hline & $\begin{array}{c}\text { Nenhum } \\
\text { problema }\end{array}$ & $\begin{array}{c}\text { Um } \\
\text { problema }\end{array}$ & $\begin{array}{c}\text { Dois } \\
\text { problema } \\
\text { s }\end{array}$ & $\begin{array}{c}\text { Três ou } \\
\text { mais } \\
\text { problema } \\
\text { s }\end{array}$ & $\boldsymbol{F}$ \\
\hline $\begin{array}{l}\text { Saúde física } \\
\begin{array}{l}\text { Bem-estar emocional } \\
\text { e psicológico }\end{array}\end{array}$ & $5.80 \mathrm{a}$ & $5.23 \mathrm{~b}$ & $5.07 \mathrm{c}$ & $4.34 \mathrm{~d}$ & $34.66^{* * *}$ \\
$\begin{array}{l}\text { Atividade profissional } \\
\begin{array}{l}\text { Educação das } \\
\text { crianças/jovens }\end{array}\end{array}$ & $6.05 \mathrm{a}$ & $5.45 \mathrm{~b}$ & $5.23 \mathrm{~b}$ & $4.41 \mathrm{c}$ & $33.00^{* * *}$ \\
$\begin{array}{l}\text { Adesão às medidas } \\
\text { de confinamento e } \\
\text { isolamento social }\end{array}$ & $5.66 \mathrm{a}$ & $5.32 \mathrm{a}$ & $4.98 \mathrm{ab}$ & $4.54 \mathrm{~b}$ & $14.75^{* * *}$ \\
\hline
\end{tabular}

Nota: ${ }^{*}: p \leq 0.05 ;{ }^{* *}: p \leq 0.01 ;{ }^{* * *}: p \leq 0.001$; Letras diferentes em cada linha indicam médias significativamente diferentes.

As classes 1 e 3 , que reúnem respetivamente $24.88 \%$ e $23.98 \%$ das formas classificadas, remetem para um novo perspetivar da casa advindo de aí passar mais tempo e da importância da casa no contexto da pandemia como lugar seguro, de descanso e conforto.

2 Uma análise comparativa do impacto avaliado por quem respondeu à pergunta aberta e quem não respondeu indica que os últimos avaliaram o impacto da habitação como menos positivo na saúde física, $t(505)=1.98, p=.049$, no bem-estar emocional, $t(506)=2.03, p=.043$, e na adesão às medidas de confinamento, $t(500.75)=2.70, p=.007$. 
Estas classes de palavras são evocadas de um modo geral por todas as pessoas participantes não se encontrando associadas a nenhuma condição habitacional específica.

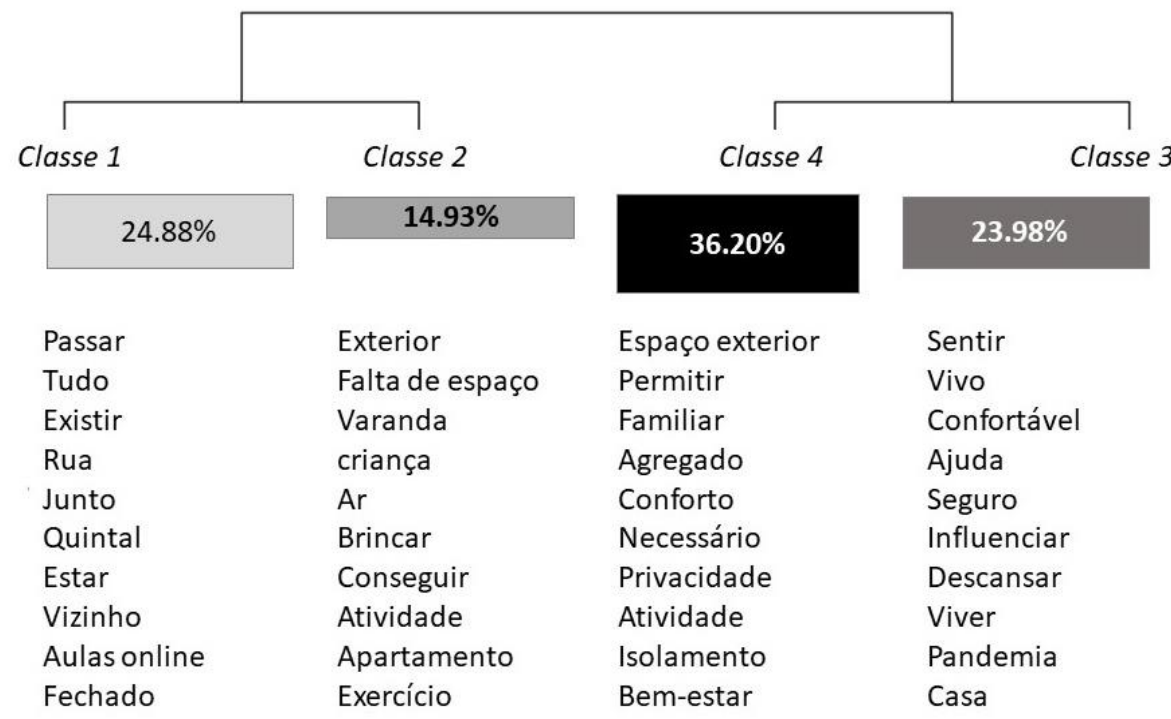

Fig. 2. Dendrograma, peso relativo das classes e formas mais significativas de cada classe.

As transcrições das respostas dadas pelas pessoas participantes ilustram algumas das mudanças perspetivadas:

Passei a ter mais tempo para organizar melhor tudo o que tenho em casa, desde de roupas que já não uso a louças e outros objetos. (apartamento arrendado, três ou mais problemas)

Passou a aproveitar-se muito mais cada dependência da casa, sobretudo a sala de estar que ganhou muito mais uso! (moradia própria sem empréstimo, um problema)

Com a pandemia passei a conhecer os vizinhos e aumentou a solidariedade. (apartamento próprio com empréstimo, nenhum problema)

Internet para trabalhar e aulas online. (moradia cedida, um problema)

llustram ainda a importância da casa como lugar seguro, espaço de conforto, descanso e descontração:

Nosso lar nosso conforto nossa segurança, e onde nos sentimos seguros nesse momento de pandemia. (moradia própria sem empréstimo, um problema)

Tem influenciado de forma positiva, uma vez que é um local onde me sinto muito confortável, é o meu espaço! (apartamento próprio com empréstimo, nenhum problema)

É um local onde me sinto bem e consigo descontrair e libertar o stress. (moradia própria sem empréstimo, nenhum problema)

No primeiro confinamento, quando estava tudo fechado e desconhecíamos mais acerca do vírus, podia circular no meu quintal, apanhar sol, respirar ar livre, o que era bom e ajudava. (moradia própria sem empréstimo, um problema)

Por seu lado, as formas classificadas na classe 2 (14.93\%) e na classe 4 (36.20\%) remetem para os impactos negativos e positivos das condições habitacionais, com especial incidência para a ausência vs. existência de espaço exterior. 
A classe 2 é mais representativa dos respondentes que vivem em apartamentos $\left(\chi^{2}=\right.$ $12.98, p<.001)$, em habitações que apresentam três ou mais problemas $\left(\chi^{2}=5.28, p<\right.$ $.05)$, cedidas $\left(\chi^{2}=9.99, p<.01\right)$ ou renda apoiada $\left(\chi^{2}=6.41, p<.05\right)$. A análise do contexto de evocação das palavras incluídas nesta classe mostra, nomeadamente, as dificuldades encontradas em conciliar as atividades profissionais com as atividades escolares e de lazer das crianças quando o espaço é reduzido e o isolamento sonoro insuficiente:

Por ser muito pequena para os quatro (tem cerca de $65 \mathrm{~m}^{2}$ ), é um grande desafio encontrar formas de gerir o espaço. Acabar uma aula, trazer a mesa para o quarto para começar uma reunião, interrupções. (apartamento próprio com empréstimo, dois problemas)

Tenho só uma secretária com PC num lugar da casa de passagem comum para os quantos, para mim é difícil trabalhar em casa quando as escolas estão fechadas com aulas online, visto que tenho que dividir o "ambiente de trabalho" com o "ambiente escolar". (moradia cedida, dois problemas)

Só havendo sala, e sendo pequena torna-se desconfortável estar eu a ter aulas online com o meu namorado e o cão na mesma divisão. A concentração nem sempre é conseguida. Há falta de espaço para fazer exercício físico. (apartamento arrendado, três ou mais problemas)

Apartamento a custos controlados, sem varandas e com mau isolamento sonoro, o que condiciona o comportamento de duas crianças de primeira infância confinadas em casa. (apartamento próprio com empréstimo, três ou mais problemas)

A falta de espaço exterior dificulta os tempos de lazer saudáveis, principalmente os das crianças. (apartamento cedido, um problema)

llustram ainda a importância do isolamento térmico e acústico, dos problemas de humidades e infiltrações e da falta de espaço para a saúde física e para o bem-estar emocional e psicológico:

E se eu ou o meu companheiro ficarmos infetados não há hipótese de distanciamento isolamento pois é um apartamento muito pequeno, apesar de ter dois quartos. A humidade que, entretanto, apareceu também não é nada boa para a saúde, sobretudo para quem teve pneumonia recentemente [...] e para mim que tenho rinite alérgica há anos. Estar confinada num sítio tão pequeno também me provoca mais ansiedade. (apartamento arrendado, três ou mais problemas).

As condições de isolamento sonoro e temperatura não são as melhores, o que influencia negativamente o teletrabalho. (apartamento arrendado, três ou mais problemas)

O fato de estar condicionado ao apartamento faz com que o humor/ bemestar oscile diariamente entre o estar bem e o estar psicologicamente exausta. Algum ruído causado pelos vizinhos é agora encarado de uma forma menos paciente. (apartamento próprio com empréstimo, nenhum problema)

$O$ frio e a humidade durante tantas semanas em que tivemos que trabalhar de casa e viver cá $100 \%$ dos dias fez-nos sentir muito mal, nada confortáveis, a pensar em mudar urgentemente de casa, humidade nas paredes, roupas. etc. Pouco sol direto, não temos jardim para poder estar em contacto com a natureza e aliviar o stress. (apartamento próprio sem empréstimo, três ou mais problemas)

Falta de privacidade, isolamento sonoro e de luz natural - tudo isto afeta o estado emocional sempre que passamos nos mesmo espaço 24/24horas. (apartamento arrendado, três ou mais problemas)

Sinto-me a viver numa prisão. (apartamento próprio com empréstimo, três ou mais problemas) 
Pelo contrário, a classe 4 é representativa das respostas dos participantes que vivem em moradias $\left(\chi^{2}=15.06, p<.001\right)$, que não apresentam problemas $\left(\chi^{2}=4.06, p<.05\right) \mathrm{e}$ constituem habitação própria adquirida com recurso a empréstimo bancário $\left(\chi^{2}=8.44, p<\right.$ .01). As respostas ilustram como a existência de espaço interior e exterior contribuem para a conciliação das diversas atividades, para a prática de exercício físico e para um melhor bem-estar físico e psicológico:

Possuir espaço exterior, não necessitar de cruzar espaço com outras famílias, e ter uma dimensão que permite se for necessário, realizar isolamento de qualquer dos membros da família. (morada própria sem empréstimo, nenhum problema)

O facto de ser moradia com espaço exterior e mundo rural tem permitido outras atividades e contacto com a natureza contribuindo para um melhor equilíbrio mental. (moradia própria sem empréstimo, um problema)

A minha casa é muito espaçosa então permite mudar de divisão para assistir a aulas online, sem ser perturbada. Permite-me fazer exercício físico e atividades de culinária, que me têm ajudado a manter ocupada nesta pandemia. (moradia própria com empréstimo, um problema).

O facto de ter espaço exterior ajuda bastante durante a pandemia. Há sempre atividades com que ocupar o tempo. Permite um contacto constante com a natureza. Permite ter bastante privacidade. (moradia cedida, um problema).

Casa espaçosa, bem iluminada, com jardim em conjunto com bens de conforto permite um melhor estado de espírito e balanço emocional, assim como espaço e motivação para a prática de exercício. (moradia própria sem empréstimo, um problema)

Tem contribuído muito para o meu equilíbrio emocional e descanso tão merecido. (moradia própria com empréstimo, nenhum problema)

\section{Conclusões}

Neste artigo procuramos explorar o impacto das condições habitacionais na vivência da pandemia da Covid-19 recorrendo a métodos mistos. Para o efeito analisamos os dados preliminares de um inquérito por questionário com perguntas abertas e fechadas aplicado em Portugal durante o segundo confinamento geral.

Os resultados obtidos através das metodologias quantitativas indicam que as diferenças nas condições de conforto das habitações (e.g. espaço interior e exterior, isolamento térmico e acústico, infiltrações, exposição solar) produzem impactos significativamente diferentes na saúde física e no bem-estar emocional e psicológico, bem como no desempenho da atividade profissional e na educação de crianças e jovens a cargo. As pessoas que vivem em habitações com menor número de problemas reportam, em todas as esferas, um impacto mais positivo da habitação na vivência das alterações provocadas pela pandemia da Covid-19.

O recurso a metodologias qualitativas permitiu identificar as dimensões representacionais da casa importantes no contexto da pandemia e o modo como influenciam a vivência das alterações por ela provocadas. Os resultados revelam que o impacto positivo da casa se associa sobretudo à possibilidade de praticar exercício físico e de desenvolver atividades de lazer ao ar livre, de contactar com a natureza, identificadas como tendo potencial de aliviar o stress, distrair e assim contribuir para uma melhor saúde mental. Pelo contrário, a falta de espaço interior ou exterior, os problemas de humidade e infiltrações, o mau isolamento sonoro e térmico ou a pouca exposição solar estão associados à experiência ou agravamento de problemas de saúde física, mas também maior irritação, exaustão, falta de concentração ou ansiedade. 
O estudo apresenta algumas limitações. Apenas cerca de metade das pessoas participantes no inquérito respondeu à questão aberta e esta omissão não foi aleatória: quem não respondeu avaliou o impacto da habitação como menos positivo do que quem respondeu. Não são também negligenciáveis as questões de desejabilidade social e de autoapresentação presentes em estudos de autorrelato e as limitações da amostra no que refere aos usuais enviesamentos em termos de nível de escolaridade e rendimento decorrentes da aplicação online (Granello, \& Wheaton, 2004). Estas limitações sugerem uma subavaliação dos impactos negativos da falta de condições habitacionais, nomeadamente o silenciamento de situações mais extremas como a exaustão parental (Roskam, Aguiar, Akgun, et al., 2021) ou a violência doméstica (Kaukinen, 2020; Kofman \& Garfin, 2020).

Contudo, e apesar das limitações, os resultados suportam a importância de utilizar métodos mistos, de combinar metodologias quantitativas e qualitativas para compreender as dimensões e significados das desigualdades e dos impactos psicossociais das condições habitacionais na vivência da pandemia da Covid-19. Enquanto as metodologias quantitativas evidenciam a magnitude das desigualdades, o recurso a metodologias qualitativas permite apreender os significados dessas desigualdades.

Os resultados revelam que as medidas de confinamento e isolamento em casa produzem vivências muito diferenciadas em função das condições habitacionais, com importantes impactos na saúde física e mental. Eles salientam, como refere Farha (2020), a importância da habitação na preservação da vida humana e a urgência de abordar as desigualdades estruturais na habitação, protegendo a função social da habitação e tornando efetivo o direito a uma habitação adequada.

\section{Agradecimentos}

Agradece-se o financiamento da Fundação para a Ciência e Tecnologia no âmbito do Programa de Estímulo ao Emprego Científico (DL 57/2016/CP1341/CT0030). Agradecese também às pessoas que participaram no inquérito e às instituições que colaboraram na sua divulgação.

\section{Referências}

Aalbers, M.B. (2016). The financialization of housing: A political economy approach. London: Routledge.

Alexander, C., Bruun, M. H., \& Koch, I. (2018). Political economy comes home: On the moral economies of housing. Critique of Anthropology, 38(2), 121-139. doi:10.1177/0308275X18758871

Farha, L. (2016). Adequate housing as a component of the right to an adequate standard of living. Retirado de http://www.unhousingrapp.org/user/pages/04.resources/Thematic-Report-4-TheRight-to-Life-and-the-Right-to-Housing.pdf

Farha, L. (2020). COVID-19 Guidance Note:Protecting renters and mortgage payers. Retirado de https://www.ohchr.org/Documents/lssues/Housing/SR_housing_COVID19_guidance_rent_and_mortgage_payers.pdf

Fernandez, R., Hofman, A., \& Aalbers, M. B. (2016). London and New York as a safe deposit box for the transnational wealth elite. Environment and Planning A, 48(12), 2443-2461.

Granello, D. H. \& Wheaton, J. E. (2004). Online data collection: Strategies for research. Journal of Counseling \& Development, 82, 387-393.

Harvey, D. (1973). Social justice and the city. Oxford: Blackwell.

Kaukinen, C. (2020). When stay-at-home orders leave victims unsafe at home: Exploring the risk and consequencesof intimate partner violence during the COVID-19 pandemic. American Journal of Criminal Justice, 45, 668-679. 
Kofman, Y. B., \& Garfin, D. R. (2020). Home is not always a haven: The domestic violence crisis amid the COVID-19 pandemic. Psychological Trauma: Theory, Research, Practice, and Policy, 12(S1), S199-S201. https://doi.org/10.1037/tra0000866

ONU (2020). Organização Mundial da Saúde declara novo coronavírus uma pandemia. Retirado de https://news.un.org/pt/story/2020/03/1706881

Ramos, M. G., Lima, V. M. R., \& Amaral-Rosa, M. P. (2018). Contribuições do software IRAMUTEQ para a Análise Textual Discursiva. A.P. Costa, D. Neri de Souza, P. A. Castro, R. A. Saavedra, \& S. Oliveira e Sá (Eds.), Atas do $7^{\circ}$ Congresso Ibero-Americano em Investigação Qualitativa, 505-514.

Ratinaud, P. (2009). IRAMUTEQ: Interface de R pour les Analyses Multidimensionnelles de Textes et de Questionnaires [software]. Retirado de http://www.Iramuteq.org

Reinert, M. (1990). ALCESTE une méthodologie d'analyse des données textuelles et une application: Aurélia de Gerard de Nerval. Bulletin de Méthodologie Sociologique, 26(1), 2454.

Reinert, M. (1993). Les "mondes lexicaux" et leur "logique" à travers l'analyse statistique d'un corpus de récits de cauchemars. Langage et Société, 66, 5-39.

Ribeiro, R., \& Santos, A. C. (2019). Financeirização da habitação e desigualdades socioterritoriais: um estudo comparado das Áreas Metropolitanas de Lisboa e do Porto. Análise Social, 233(4), 726-758.

Ribeiro, R., Santos, A.C., Poeschl, G., Jesus, F., Coelho, L., Campos, R., Silva, R., Holz, S., \& Castela, T. (2021). Pandemia da Covid-19 e habitação: Resultados preliminares. In Comunicação apresentada no Dia do Neces, Centro de Estudos Sociais, Universidade de Coimbra.

Rolnik, R. (2013). Late neoliberalism: The financialization of homeownership and housing rights. International. Journal of Urban and Regional Research, 37(3), 1058-1066.

Roskam, I., Aguiar, J., Akgun, E. et al. (2021). Parental burnout around the globe: A 42-Country study. Affective Science, 2, 58-79.

Santos, A.C. (Coord.) (2019). A nova questão da habitação em Portugal: Uma abordagem de Economia Política. Coimbra: Actual Editora. 